\title{
Magnetic helicity injection in NOAA 11261 associated with flares
}

\section{Haiqing $\mathrm{Xu}^{1}$, Hongqi Zhang ${ }^{1}$, Jiangtao $\mathrm{Su}^{1}$, Guiping Ruan ${ }^{2}$ and Jihong liu ${ }^{3}$}

${ }^{1}$ Key Laboratory of Solar Activity, National Astronomical Observatories, Chinese Academy of Sciences, Beijing 100012, China, email: xhq@bao.ac.cn

${ }^{2}$ Shandong Provincial Key Laboratory of Optical Astronomy and Solar-Terrestrial Environment, Shandong University at Weihai, Weihai 264209, China, email: rgp@sdu.edu.cn

${ }^{3}$ Department of physics electrical engineering department of information, Shi Jiazhuang University, Shi Jiazhuang 050035, China, email: liujh@bao.ac.cn

Magnetic helicity was found important in understanding solar activities such as flares and coronal mass ejections (CME). Berger and field (1984) derived an expression for helicity flux $d H_{m} / d t$, that can be applied to an individual solar active region (AR) occupying an area $S$ of the photosphere,

$$
d H_{m} / d t=-2 \int_{s}\left[\left(\mathbf{A}_{\mathbf{p}} \cdot \mathbf{V}\right) \mathbf{B}-\left(\mathbf{A}_{\mathbf{p}} \cdot \mathbf{B}\right) \mathbf{V}\right] d S,
$$

where $\mathbf{A}_{\mathbf{p}}$ is the vector potential of potential field, and $\mathbf{V}$ is the plasma velocity at the surface $S$. The first term describes the effect of magnetic footpoint motions on the surface $S$. The second term describes the flux of helicity advected through the surface when already twisted and/or writhed flux ropes emerge. Chae (2001) proposed a method of self-consistently determining magnetic helicity injection rate, $d H / d t$, using a time series of longitudinal magnetograms only:

$$
d H / d t=-\int 2\left(\mathbf{A}_{p} \cdot \mathbf{V}_{L C T}\right) B_{n} d S
$$

where $B_{n}$ is the normal component of magnetic field. $A_{p}$ is the vector potential computed from $B_{n}$ by Fourier transform method. $\mathbf{V}_{L C T}$ is the horizontal component of velocity determined by the technique of local correlation tracking (LCT). This technique was applied by some scientists (e.g., Chae et al., 2001; Nindos and Zhang, 2002; Romano et al., 2003). Magnetic helicity injection was found to be strongly correlated with the occurrence of major flares (Moon et al. 2002a, 2002b; Park et al., 2008; Labonte et al., 2007; Maeshiro et al., 2009).

In this paper, we use equation (2) to compute the magnetic helicity injection rate. We use the full-disk longitudinal magnetograms with a cadence of 12-min of the Helioseismic and Magnetic Imager (HMI; Schou et al., 2012) onboard the Solar Dynamics Observatory (SDO) from 00:00 UT on 2011 July 29 to 23:48 UT on August 4 . The full-disk magnetograms are recorded by a $4096 \times 4096 \mathrm{CCD}$ with a pixel size of $0.5^{\prime \prime}$. We choose a $450^{\prime \prime} \times 280^{\prime \prime}$ region of magnetogram covering the whole AR NOAA 11261 to analyze. The reference time is 00:00 UT on 2011 August 2, when the AR passed through the central meridian. The nonlinear mapping and the geometrical foreshortening correction all have been done following the method by Chae et al. (2001).

This AR is a $\beta$ - type sunspot on July 29 . During the period of our study, the positive polarity of main spots gradually declined and almost disappeared on August 2, and the negative polarity expanded, small bipolar spots emerged to the west of the main negative polarity from July 30. The new magnetic flux continued emerged and interacted with the 

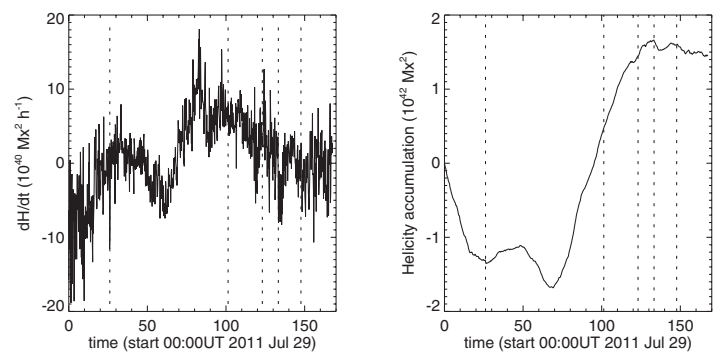

Figure 1. Left: Helicity transport rate as a function of time. Right: Accumulated change of the magnetic helicity. The time unit is hour. The dashed lines represent the time of M- class flares.

old magnetic flux, the AR became more and more complex. There was a strong shear along the inverse line between the old negative polarity and the new positive polarity. There were total 5 M-class and 7 C-class flares erupting in this AR.

From the left panel of figure 1, we can see that at first, the amplitude of helicity transport rate $d H / d t$ was negative, then decreased in magnitude. From August 1 the positive $d H / d t$ increased and reach to the maximum around 11:00 UT, then decreased smoothly to around zero. The right panel shows that there is large of negative helicity accumulated before the first M9.3 (July 30, 02:04 UT) flare erupted. After reaching to peak of about $-1.68 \times 10^{42} M x^{2}$ in about 69 hours, it then changed the sign and fast increased to the peak of about $1.66 \times 10^{42} M x^{2}$ at 13:00 UT on August 3. In this phase, there were 3 M-class flares erupting. After the last M9.3 (August 4, 03:41 UT) flare erupted, the accumulated change of magnetic helicity become steadily.

Based on the above analysis, we summarize that: (1) The negative magnetic helicity was transported before the first M9.3 flare erupted and positive magnetic helicity was transported when the later $4 \mathrm{M}$ - class flares erupted. (2) Except for M1.4, the helicity accumulation were all reached to $1 \times 10^{42} M x^{2}$ before the M- class flares erupted. (3) There was a strong shear along the magnetic neutral line which may be one mechanism for triggering the flares. In the flare erupting region, the helicity transport rate is relatively large which may also trigger the flares.

This work is supported by the National Natural Science Foundation of China (Grant Nos. 10978015, 11003025, 11178016, 41174153, 10921303, 11178005, 11125314, 11103037, 11103038), Knowledge Innovation Program of The Chinese Academy of Sciences (Grant No. KJCX2-EW-T07), the Young Researcher Grant of National Astronomical Observatories, Chinese Academy of Sciences.

\section{References}

Berger, M. A. \& Field, G. B. 1984, J. Fluid Mech., 147, 133.

Chae, J. 2001, ApJ, 560, L95.

Chae, J., Wang, H., Qiu, J., Goode, P. R., Strous, L., \& Yun, H. S. 2001, ApJ, 560, 476.

Labonte, B. J., Georgoulis, M. K., \& Rust, D. M. 2007, ApJ, 671, 955.

Maeshiro, T., Kusano, K., Yokoyama, T., \& Sakurai, T. 2005, ApJ, 620, 1069.

Moon, Y.-j., Chae, J., Choe, G. S., et al. 2002a, ApJ, 574, 1066.

Moon, Y.-j., Chae, J., Wang, H., Choe, G. S., \& Park, Y. D. 2002b, ApJ, 580, 528.

Nindos, A. \& Zhang, H. 2002, Astrophys. J. Lett. 573, L133.

Park, S.-H., Lee, J., Choe, G. S., et al. 2008, ApJ, 686, 1397.

Romano, P., Contarino, L., \& Zuccarello, F. 2003, Sol. Phys., 218, 137.

Schou, J., Borrero, J. M., \& Norton, A. A., et al. 2012, Sol. Phys., 275, 327. 